\title{
TITLE:
}

\section{New Addition Reactions. (IV) : The Addition of Schiff Bases to Diketene}

\section{AUTHOR(S):}

Oda, Ryohei; Takashima, Shunichi; Okano, Masaya

\section{CITATION:}

Oda, Ryohei ...[et al]. New Addition Reactions. (IV) : The Addition of Schiff Bases to

Diketene. Bulletin of the Institute for Chemical Research, Kyoto University 1963, 41(2-4): 223-223

ISSUE DATE:

1963-10-19

URL:

http://hdl.handle.net/2433/75956

RIGHT: 


\begin{abstract}
S
(2) In the decarboxylation of 3-aryl-2-oxazolidinones, the electronic effect of the substituent on the benzene ring indicates that the fission of the $\mathrm{N}-\mathrm{C}$ bond of the urethane group in the 2-oxazolidinone ring is rate-determining. (3) The initial rate of the aliphatic amine-catalyzed decarboxylation of 3-aryl-2-oxazolidinones is of the pseudo-first-order; it also depends on the concentration and structure of the amine. A mechanism has been proposed which involves a nucleophilic attack of amine on the carbonyl-carbon atom. An amine having an hydroxyl or amino group on the $\beta$-carbon shows a large rate-acceleration; this suggests the simultaneous electrophilic participation by the active hydrogen of these groups. (4) The initial rate of the decarboxylation of 2-oxazolidinone itself, catalyzed by aliphatic amine, is of the second-order in oxazolidinone and of the first-order in amine. From this a concerted mechanism has been inferred, in which the amine acts as a nucleophile and the other oxazolidinone acts as an electrophile.
\end{abstract}

New Addition Reactions. (IV)

\title{
The Addition of Schiff Bases to Diketene
}

\author{
Ryohei Oda, Shunichi Tarashima and Masaya Okano \\ Blletin of the Chemical Society of Japan, 35, 1843 (1962)
}

Treatment of diketene with $\mathrm{N}$-benzylidene-t-butylamine afforded a cyclic adduct, adduct, 1-t-butyl-6-phenyl-2,4-piperidinedione, in an excellent yield. Simlar reactions with $\mathrm{N}$-methylene-t-butylamine and $\mathrm{N}$-methylenecyclohexylamine also gave the corresponding piperidinediones, but the yields were very low.

\section{New Addition Reactions. (V) Dimerization of Diketene}

\author{
Ryohei Oda, Shunichi Takashima and Masaya Okano
}

Bulletin of the Chemical Socity of Japan, 36, 476 (1963)

When diketene was treated with $5-10 \mathrm{~mol} . \%$ of aluminum tribromide in ethylene dichloride at $10-20^{\circ} \mathrm{C}$ for about thirty hours, a mixture of two dimers, 2,6-dimethyl- $\gamma$-pyrone-3-carboxylic acid and dehydroacetic acid (ratio 4:7), was obtained in ca. $60 \%$ yield without any formation of a polymeric by-product. This result is contrast to other Lewis acid-catalyzed reactions (cf, Makromol. Chem., 39, 243 (1960); 43, 149 (1961)). 